\title{
Electrical Stimulation of the Human Descending Motor Tracts at Several Levels
}

\author{
Yoshikazu Ugawa, Kieko Genba-Shimizu and Ichiro Kanazawa
}

\begin{abstract}
The descending motor tracts were activated using a high voltage electrical stimulation technique at four levels: the motor cortex, brainstem (around the pyramidal decussation), and the first and sixth thoracic vertebral levels (T1, T6). Electromyographic activity was recorded from the tibialis anterior or extensor digitorum brevis muscles. Consistent motor action potentials could be evoked in all normal subjects. The conduction velocity of the descending tracts was estimated to be 62 to $79 \mathrm{~m} / \mathrm{s}$. The sum of synaptic delay and utilization time at the motoneurons (spinal delay time) was estimated to be $0.5 \pm 0.3 \mathrm{~ms}$ indicating a mono- or oligosynaptic connection. In patients with diffusely affected descending tracts, the technique showed slowed conduction along the descending tracts and prolonged spinal delay time which may include the time required for temporal summation of excitatory postsynaptic potentials as well as synaptic delay and utilization time. In patients with a localized lesion, localized conduction delay was found at the appropriate segment.
\end{abstract}

\begin{abstract}
RÉSUMÉ: Stimulation électrique à plusieurs niveaux des faisceaux moteurs descendants chez l'humain. Nous avons activé les faisceaux moteurs descendants, par une technique de stimulation électrique à haut voltage, à quatre niveaux: au niveau du cortex moteur et du tronc cérébral (autour de la décussation pyramidale), et au niveau de la première et de la sixième vertèbre thoracique (D1, D6). Nous avons enregistré l'activité électromyographique du jambier antérieur ou du muscle pédieux. Chez tous les sujets normaux, des potentiels moteurs d'action pouvaient être évoqués de façon constante. La vitesse de conduction des faisceaux descendants a été estimée comme étant de 62 à $79 \mathrm{~m} / \mathrm{s}$. La somme du délai synaptique et du temps d'utilisation au niveau des motoneurones (délai spinal) a été estimée à $0.5 \pm 0.3 \mathrm{~ms}$, indiquant qu'il s'agit d'une connexion mono ou oligosynaptique. Chez les patients qui ont une pathologie diffuse des faisceaux descendants, cette technique a montré une conduction ralentie au niveau des faisceaux descendants et un délai spinal prolongé qui peut inclure le temps requis pour la sommation temporelle des potentiels post-synaptiques excitateurs ainsi que le délai synaptique et le temps d'utilisation. Chez les patients qui ont des lésions localisées, nous avons observé un délai de conduction localisé au niveau du segment approprié.
\end{abstract}

Can. J. Neurol. Sci. 1995; 22: 36-42

It is now possible to study human central motor pathways using electrical and magnetic stimulation techniques. ${ }^{1}$ Magnetic stimulation is virtually painless and is now widely used. However, present magnetic stimulators are only capable of exciting the cerebral motor cortex in the skull and the spinal nerve roots as they exit the intervertebral foramen. As yet they are unable to activate directly structures within the spinal cord itself. ${ }^{1,2}$ High-voltage percutaneous electrical stimulation is less comfortable for the subject, but in contrast can be used to excite the motor pathways at almost all levels from the cortex to the spinal cord. ${ }^{3-5}$ In the present paper, we show how this can be used to detect lesions of descending motor tracts in the spinal cord.

\section{SubJECTS}

We studied 14 patients (25-60 yrs of age; 10 males and 4 females: $155-172 \mathrm{~cm}$ tall) who had abnormalities of the descending motor tracts in the spinal cord. A 32-yr-old woman with Friedreich's ataxia (Patient 1) showed bilateral pyramidal and posterior columnar signs which had begun in childhood. No structural abnormalities were present in the magnetic resonance imaging (MRI) scan of her brain and spinal cord. The diagnosis of the patient with adrenoleukodystrophy (Patient 2) was based on the elevation of long chain fatty acids in the serum. The diagnosis for four patients (Patients 3-6) with a localized structural lesion compressing the spinal cord was confirmed by MRI and myelography. A 25-yr-old man (Patient 7) had spastic paraparesis and an atonic bladder. His MRI scan revealed one plaque continuing from the seventh cervical to the third toracic vertebral level, which disappeared after predonisolone treatment. In another patient with multiple sclerosis (Patient 8), the MRI scan

From the Department of Neurology, Institute for Brain Research, School of Medicine, University of Tokyo, Tokyo.

RECEIVED DECEMBER 3,1993. ACCEPTED IN FINAL FORM JULY 25, 1994

Reprint requests to: Dr. Y. Ugawa, Department of Neurology, Institute for Brain

Research, School of Medicine, University of Tokyo, 7-3-1, Hongo, Bunkyo-ku, Tokyo 113 , Japan. 
showed multiple plaques in the cerebral white matter, brainstem, and spinal cord. Six patients had idiopathic spastic paraparesis with a duration of more than five years. Laboratory tests showed no vitamin deficiencies, abnormality of long chain fatty acids, or positive anti-HTLV- 1 antibody titers in any of them and the MRI showed no structural abnormalities. To obtain normal values, we studied eight healthy volunteers (27-40 yrs of age; 6 males and 2 females; $158-178 \mathrm{~cm}$ tall). All the subjects gave their informed consent. The procedure was approved by the Ethics Committee of the University of Tokyo.

\section{Methods}

In all subjects, surface electromyographic (EMG) activities were recorded from the tibialis anterior (TA) and extensor digitorum brevis (EDB) muscles on the right side. In some subjects, responses also were recorded on the left. EMG signals were amplified with filters set at $80 \mathrm{~Hz}$ and $3 \mathrm{kHz}$. The descending motor tracts or the motor roots were stimulated at several levels using a high voltage electrical stimulator as described elsewhere. ${ }^{6}$

We used special electrodes for stimulation in most experiments. They had a larger contact area $\left(7.1 \mathrm{~cm}^{2}\right)$ than conventional electrodes $\left(2.0 \mathrm{~cm}^{2}\right)$ in order to reduce pain caused by high current density in the skin. During the examination, an electrocardiogram was taken and blood pressure was monitored to check the effect of stimulation on the heart. No arrhythmia occurred after stimulation. Blood pressure and heart rate increased after stimulation, but returned to the original values within $20 \mathrm{sec}-$ onds. No side effects were noted in any of the individuals. All the participants regarded motor root stimulation to be no more uncomfortable than peripheral nerve stimulation, and regarded brainstem stimulation as being the most uncomfortable. All of the normal controls had experienced brainstem stimulation with the conventional electrodes, and reported that stimulation with new electrodes was less uncomfortable than stimulation with the conventional electrodes. All subjects tolerated the procedure.

For cortical stimulation, the anode was placed at the vertex and the cathode $5 \mathrm{~cm}$ anterior to the anode. For activation of the descending tracts at the level of pyramidal decussation ${ }^{3,4}$ the stimulating electrodes were placed on the incisura mastoidea on both sides. The descending tracts were stimulated at two levels within the spinal cord: upper thoracic level, with the cathode on the first thoracic spinous process (Sp[T1]), and the anode $5 \mathrm{~cm}$ above it, and mid-thoracic level, with the cathode on the sixth thoracic spinous process (Sp[T6]), and the anode $5 \mathrm{~cm}$ above it. The motor roots were activated at the level of the conus medullaris ${ }^{7}$ with the cathode placed at the first lumbar spinous process $(\mathrm{Sp}[\mathrm{L} 1])$ and the anode $5 \mathrm{~cm}$ above it. They were activated at the point of exit from the spinal canal using the cathode placed at the fourth lumbar spinous process (Sp[L4]) for the TA muscle and at the first sacral spinous process $(S p[S 1])$ for the EDB muscle, with the anode being placed $5 \mathrm{~cm}$ above the cathode. The muscles studied were activated during stimulation of the motor cortex or descending tracts, but relaxed during activation of the motor roots.

Total peripheral conduction time in each of the normal subjects and in some patients was estimated for each muscle using the conventional F-wave method. Total peripheral latency was calculated from the formula $(\mathrm{M}+\mathrm{F}-1) / 2(\mathrm{M}$ and $\mathrm{F}$ : latency of $\mathrm{M}$ and F-waves). ${ }^{8}$

The time required for the nerve impulses to be conducted from the motor cortex down to the three distal activation sites in the brainstem and spinal cord was calculated from the difference in latency between cortical stimulation and the stimulation at the other sites. In order to estimate conduction velocity of the stimulated descending motor tracts, we used average lengths of brain and spinal cord in postmortem specimens, ${ }^{9,10}$ because there is no reliable way to measure the lengths in intact subjects. Conduction time along the motor roots in the cauda equina also was estimated from the latency difference between stimulation over $\mathrm{Sp}[\mathrm{L} 1]$ and $\mathrm{Sp}[\mathrm{L} 4$ or S1].

The following five conduction times were calculated from the difference in latency between two sites: cortico-brainstem conduction time (CTX-BST CT), brainstem to first thoracic spinous process conduction time (BST-Sp[T1] CT), first to sixth thoracic spinous process conduction time (Sp[T1]-Sp[T6] CT), sixth thoracic to first lumbar spinous process conduction time (Sp[T6]-Sp[L1] CT), and first lumbar to fourth lumbar (or first sacral) spinous process conduction time (Sp[L1]-Sp[L4 or S1] $\mathrm{CT}$ ). The first three indicate conduction times along the descending tracts, and Sp[L,1]-Sp[L4 or S1] CT indicates the conduction time along the motor roots in the cauda equina. Sp[T6]-Sp[L1] CT may include the conduction times along the descending tracts and along the motor roots in the spinal canal, as well as the utilization time and synaptic delay at the motoneurons, and the time required for temporal summation of EPSPs of the descending motor tracts in some patients. The conduction times in patients were considered to be prolonged if they exceeded the upper limit of the normal range (mean $+2.5 \mathrm{SD}$ ).

\section{Results}

\section{Normal subjects}

Consistent motor responses were obtained from all the normal subjects. The thresholds for muscle activation ranged from 200 to $400 \mathrm{~V}$. Representative responses to stimulation at six levels in the EDB and TA muscles of a single subject are shown in Figure 1. In the EDB muscle (Figure 1A), the shapes of the EMG responses to brainstem and spinal cord stimulation were simple and biphasic, and very similar to the shapes of the responses elicited by activation of the motor roots. In contrast, the response to motor cortical stimulation was polyphasic and of longer duration. Very similar results were found for the TA muscle (Figure 1B). The conduction times (CTX-BST CT, BSTSp[T1] CT, Sp[T1]-Sp[T6] CT, Sp[T6]-SpL1] CT) were not different between the two muscles. The $\mathrm{Sp}[\mathrm{L1} 1]-\mathrm{Sp}[\mathrm{S} 1] \mathrm{CT}$ of the EDB was longer than the Sp[L1]Sp[L4] CT of the TA. The average values of all the conduction times of normal subjects are given in Table 1. The Sp[L1]-Sp[S1] CT of the EDB was significantly $(p<0.01)$ longer than the Sp[L1]-Sp[L4] CT of the TA. There were no significant differences $(p>0.1)$ between the EDB and TA muscles for the other conduction times.

In every normal subject, we estimated the conduction velocity of the stimulated descending motor tracts and motor roots by linear regression analysis between the TA conduction times and the lengths of the descending motor tracts (Figure 2). With bipolar stimulation the electrical current density is concentrated beneath the electrodes. The outward current is highest beneath the cathode and activation of nerve axons usually occurs at this point. On the assumption that activation took place beneath the cathode during spinal and motor root stimulation, we estimated the distance between the two activation sites from the average length of postmortem specimens beneath the cathode. $\mathrm{Sp}[\mathrm{T} 1]$ and $\mathrm{Sp}[\mathrm{T} 6]$, respectively, are assumed to be the T3 and T8 spinal 

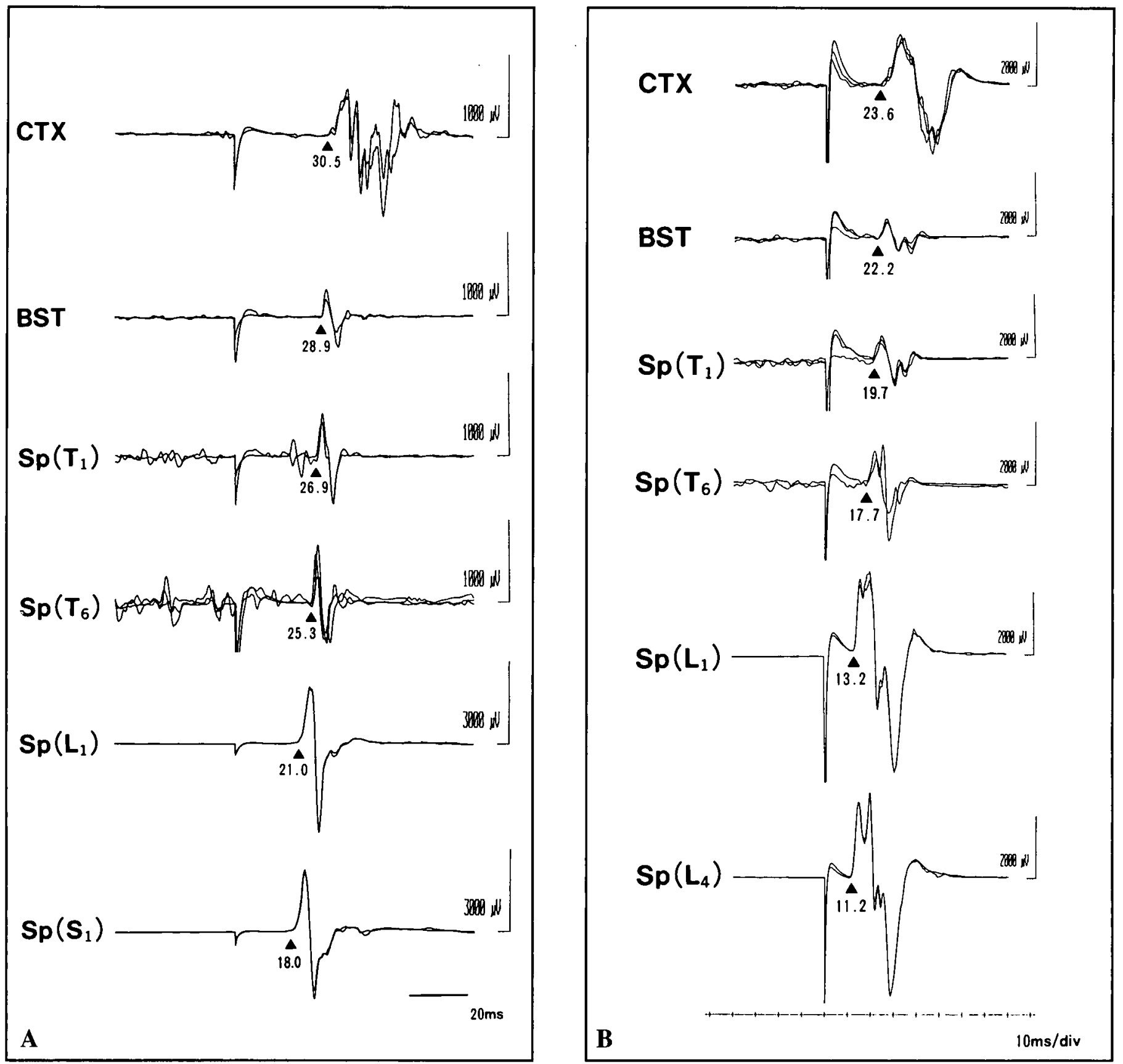

Figure 1: Responses recorded from the extensor digitorum brevis $(E D B)(A)$ and tibialis anterior $(T A)$ muscles $(B)$ of a normal subject.

The shapes of the motor action potentials elicited by brainstem (BST) and spinal cord stimulation (Sp[TIJ and Sp[T6]) are very similar to those of the responses evoked by activation of the motor roots. In contrast, cortical stimulation provoked polyphasic responses of longer duration. The differences in latency between two adjacent stimulation sites are the same for the two muscles, except for the conduction time along the motor rots, which is longer for EDB.

CTX: cortical stimulation

BST: Brainstem stimulation (Ugawa et al. [3])

Sp[T1]: stimulation at the level of the first thoracic spinous process

Sp[T6]: stimulation at the level of the sixth thoracic spinous process
Sp[LI]: stimulation at the level of the first lumbar spinous process Sp[L4]: stimulation at the level of the fourth lumbar spinous process Sp [S1]: stimulation at the level of the first sacral spinous process.

Table 1. Normative data on conduction times (ms)

\begin{tabular}{cccccc}
\hline Muscle & CTX-BST & BST-Sp(T1) & Sp(T1)-Sp(T6) & Sp(T6)-Sp(L1) & Sp(L1)-Sp(L4 or S1) \\
\hline TA & $1.6 \pm 0.3$ & $2.5 \pm 0.3$ & $1.4 \pm 0.2$ & $3.9 \pm 0.7$ & $2.0 \pm 0.3$ \\
EDB & $1.8 \pm 0.5$ & $2.6 \pm 0.4$ & $1.5 \pm 0.2$ & $4.2 \pm 0.6$ & $3.0 \pm 0.4$ \\
\hline
\end{tabular}

The mean \pm standard deviation is given for the results of eight normal subjects.

TA: tibialis anterior, EDB: extensor digitorum brevis 
cord levels." The latencies from the cortex were calculated by subtracting the latency of the response elicited by stimulation over the point of interest from the cortical latency. The latency from the cortex to the L4 spinal cord level was obtained by subtracting (M+F-1)/2 from the cortical latency. The graph was drawn as the latency from the motor cortex, shown on the abscissa, and the length from the L4-L5 foramen on the ordinate (Figure 2). From the slope of the regression line $(r=0.97-0.99)$, we estimated the conduction velocity of the descending motor tracts to be 62 to $79 \mathrm{~m} / \mathrm{s}$ (mean \pm SD: $68 \pm 5 \mathrm{~m} / \mathrm{s}$ ). That of the motor roots was 31 to $50 \mathrm{~m} / \mathrm{s}$ (mean $\pm \mathrm{SD}: 40 \pm 7 \mathrm{~m} / \mathrm{s}$ ). The time difference between two regression lines at the spinal segment (L4) was calculated in all normal subjects. It ranged in different subjects between 0.2 and 0.9 ms (mean \pm SD: $0.5 \pm 0.3$ ). This value approximates time difference between arrival of the first descending volley and activation of the spinal motoneurons. In this paper we define this value as "spinal delay time", which must include time required for temporal summation of descending volleys, synaptic delay, and utilization time. In normal subjects' active muscles, however, it does not include the time for temporal summation because the first descending volley elicits EMG responses. The present results thus suggested that the sum of synaptic delay and utilization time at spinal motoneurons was $0.2-0.9 \mathrm{~ms}$ in normals. Similar results were obtained for the EDB muscle.

\section{Patients}

Responses recorded from the right EDB muscle of a 35-yearold woman with Friedreich ataxia (Patient 1) are shown in Figure 3. The EMG activities elicited by motor root stimulation had normal shapes, and the $\mathrm{Sp}[\mathrm{L} 1]-\mathrm{Sp}[\mathrm{S} 1] \mathrm{CT}$ was within the normal range. The responses evoked by brainstem or spinal cord stimulation, however, were small and polyphasic, as compared to the simple, biphasic responses of the normal subjects. The latency differences between the responses to stimulation at two sites in the descending tracts were longer than the normal values, indicative of slow conduction in the central motor tracts.

The relationship between the conduction times of the EDB muscle in this patient and the length of the descending tracts is shown in Figure 4. Linear regression analysis showed that conduction time along the central motor pathways was highly correlated with the length of the descending tracts $(r=0.98)$, indicating that conduction in these tracts is similarly affected at all levels. The estimated conduction velocity of the descending tracts was abnormally slow $(28 \mathrm{~m} / \mathrm{s}$ ). Conduction in the motor roots (conduction velocity: $38 \mathrm{~m} / \mathrm{s}$ ) was within the normal range. The spinal delay time at the S1 motoneurons was estimated to be $3.7 \mathrm{~ms}$.

Responses from the EDB muscle of a patient with ossification of the yellow ligament (OYL) at the fourth thoracic (T4) vertebral level (Patient 4) are shown in Figure 5. No brainstem stimulation was done on this patient because the lesion was suspected to be below the T1 vertebral level and the CTX-Sp[T1] $\mathrm{CT}$ was within the normal range. Only the $\mathrm{Sp}[\mathrm{T} 1]-\mathrm{Sp}[\mathrm{T} 6] \mathrm{CT}$ was abnormally prolonged $(6.5 \mathrm{~ms})$. This suggests that conduction in the descending motor tracts was delayed somewhere between the $\mathrm{T} 1$ and $\mathrm{T} 6$ vertebral levels. We presume that compression of the spinal cord by OYL at the T4 vertebral level was the cause of the localized conduction delay in this patient.

Results for the 14 patients are given in Table 2. All the conduction times along the descending tracts were prolonged in the
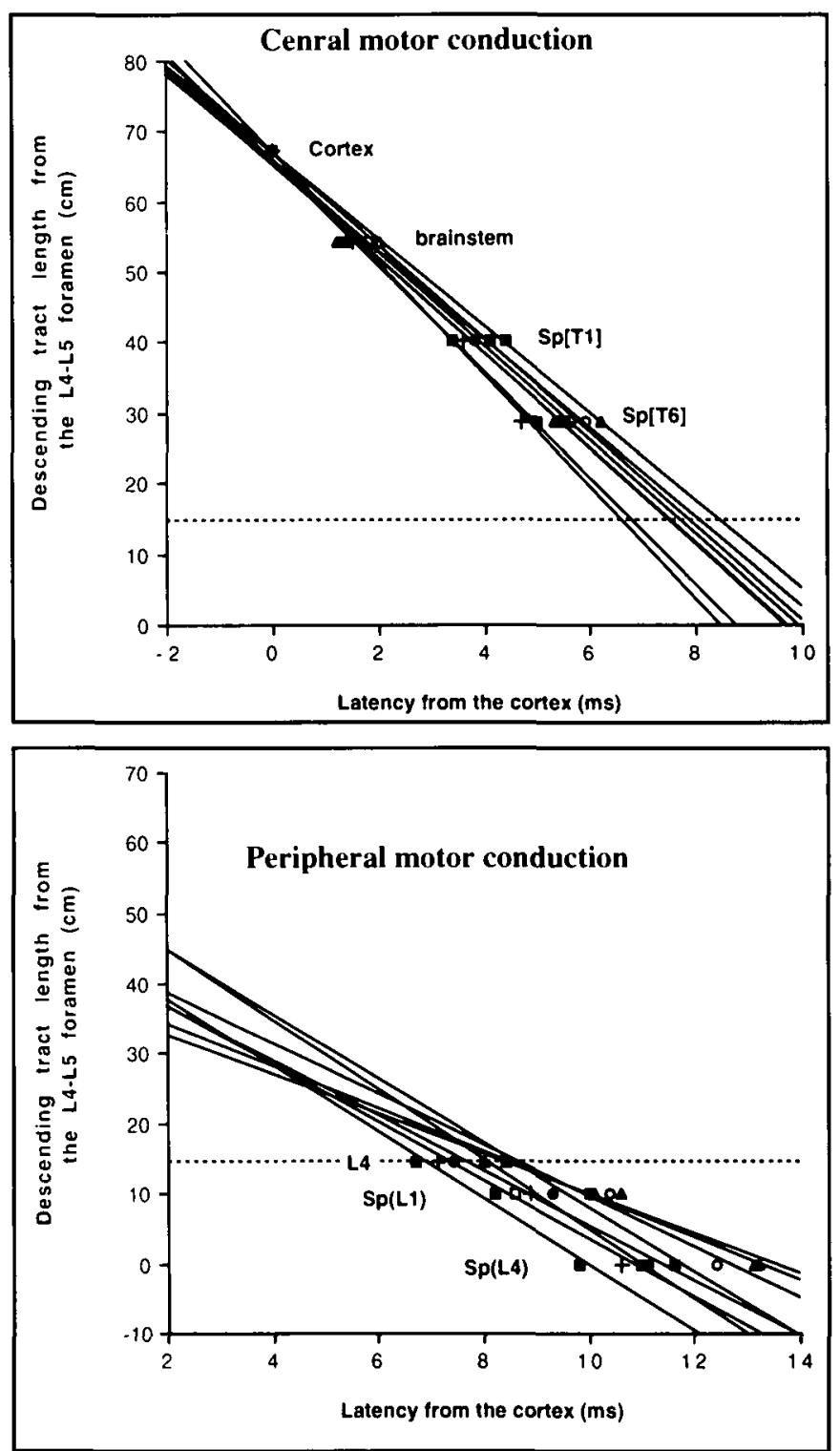

Figure 2: Relationship between the conduction times to the TA muscles and the lengths of the descending tracts.

Results of all normal subjects are superimposed. Central motor conduction is shown in the upper figure, and peripheral motor conduction in the lower. Dotted line indicates the estimated L4 spinal cord level in both figures. The difference between cortical latency and the latency of the response recorded at each stimulation site is plotted against the lengths of the descending tracts estimated from postmortem specimens. ${ }^{9.10}$ The values on the abscissa at LA (principal spinal cord level innervating $T A)$ were obtained by subtracting $(M+F-1) / 2$ from the cortical latency. The mean latency is highly correlated with the lengths of the descending tracts $(r=0.97-0.99)$. From the slope of the regression lines, conduction velocity was estimated to be 62 to $79(68 \pm 5) \mathrm{m} / \mathrm{s}$ for central motor conduction, and 31 to $50(40 \pm 7) \mathrm{m} / \mathrm{s}$ for peripheral motor conduction (motor roots). The time differences between the two lines at the L4 spinal cord level (spinal delay time) were 0.2 to $0.9 \mathrm{~ms}(0.5 \pm 0.3)$.

Abbreviations are the same as in Figure 1.

two patients with diffusely affected descending tracts (Patients $1,2)$. Of the five patients with one localized lesion (Patients 37), central motor conduction was delayed at a level consistent with a lesion in four of them (Patients 3, 4, 5 and 7). In Patient 


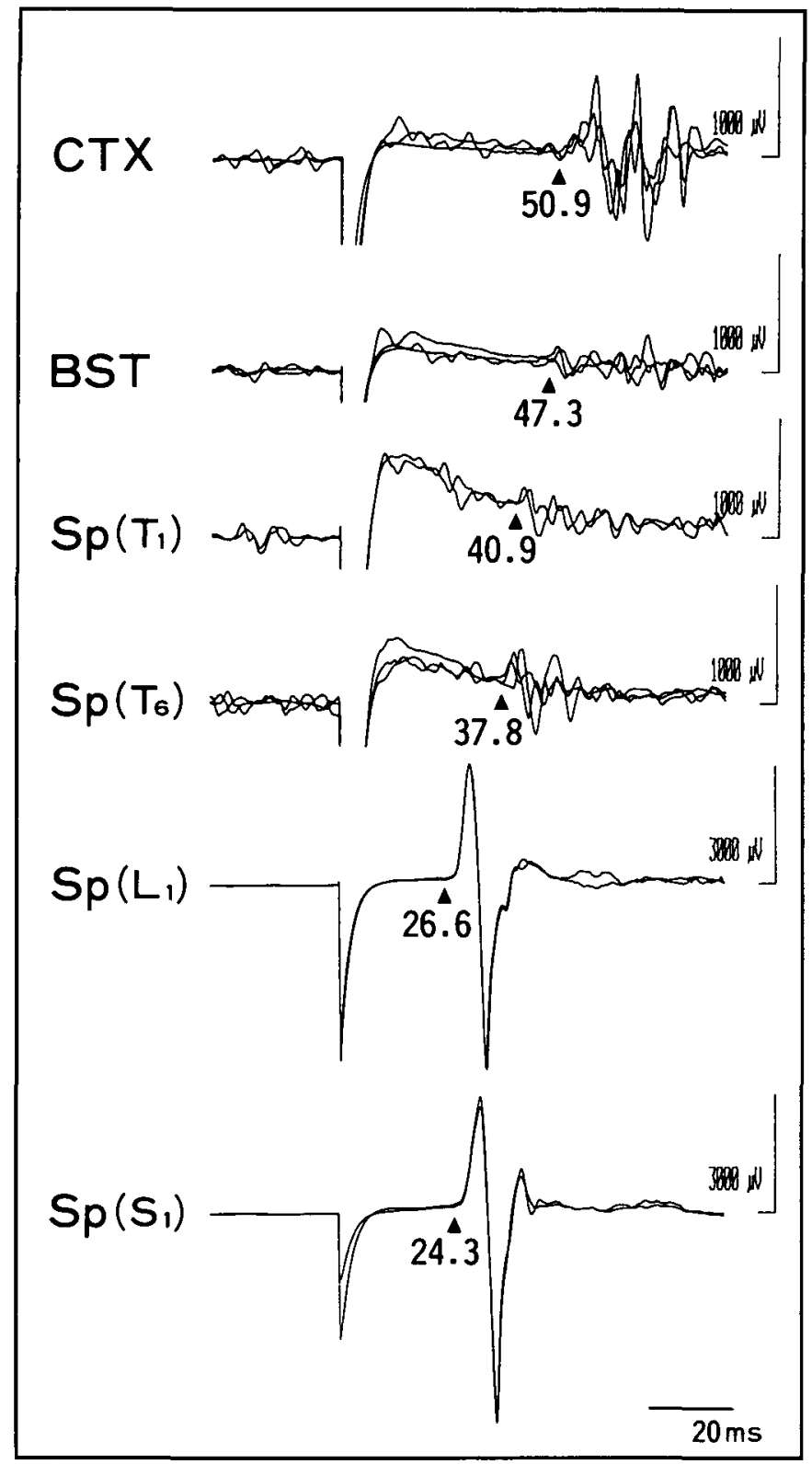

Figure 3: Responses recorded from the EDB muscle of a patient with Friedreich ataxia (Patient l).

Motor action potentials produced by activation of the descending tracts are small, of long duration, and polyphasic. All the conduction times along the descending tracts are prolonged although $S p[L 1]$-Sp[SI] CT is within the normal range.

Abbreviations are the same as in Figure 1.

7, the MRI showed that there was a plaque in the region of the corticospinal tract, which continued from the $\mathrm{C} 7$ to $\mathrm{T} 3$ vertebral level. The patient with spinal arachnoid cyst at the $\mathrm{T} 3$ vertebral level (Patient 6), had delayed $\mathrm{Sp}[\mathrm{T} 6]-\mathrm{Sp}[\mathrm{L} 1] \mathrm{CT}$ in addition to prolonged Sp[T1]-Sp[T6] CT. Prolongation of the Sp[T6]$\mathrm{Sp}[\mathrm{Ll}] \mathrm{CT}$ in this patient may be due to axonal degeneration of the descending tract below the level of compression. One patient with multiple sclerosis (Patient 8 ) showed no response on both sides when the descending tract was activated. Of the six patients with idiopathic spastic paraparesis (Patients 9-14),

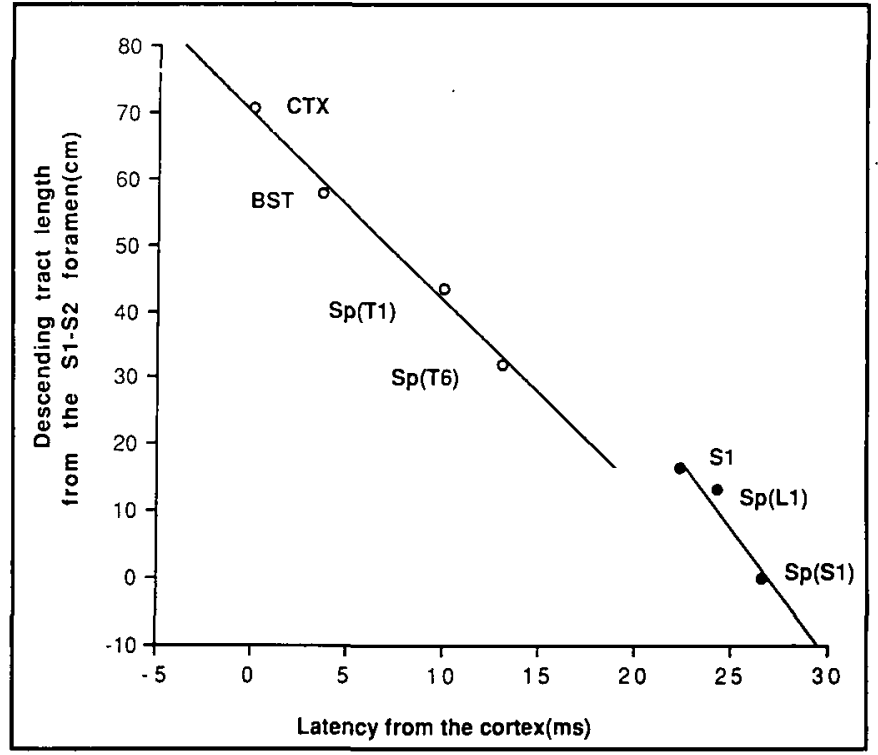

Figure 4: Regression lines for the EDB muscle responses of the patient in Figure 3 (Patient 1).

The graph is drawn in the same way as Figure 2. Central and peripheral motor conduction are plotted in the same figure. Circles represent conduction in the descending motor tracts and dots represent that in the motor roots. Latency is highly correlated with the length of the descending motor tracts. The respective estimated conduction velocities for central and peripheral motor conduction are $28 \mathrm{~m} / \mathrm{s}$ and $38 \mathrm{~m} / \mathrm{s}$.

Abbreviations are the same as in Figure 1.

S1: the first sacral spinal cord level.

central motor conduction was diffusely affected in four; although it was normal in the other two, the threshold for activating the descending tracts $(700$ to $800 \mathrm{~V}$ ) was abnormally high.

\section{Discussion}

We used high voltage electrical stimulation to activate directly long tracts within the spinal cord and produce excitation of lumbar motoneurones at short latency. Many possible tracts may be activated by this technique including corticospinal, reticulospinal, vestibulospinal, propriospinal systems. Indeed, there may even be antidromic activity in dorsal column fibers which could activate motoneurones via local reflex pathways in the lumbar spinal segments. It is not clear which, if any, of these systems is the most important in producing the results observed here. However, since neither TA nor EDB muscles have prominent monosynaptic reflexes after peripheral nerve stimulation, we think that the contribution from antidromic dorsal column activity is likely to be small. We shall therefore refer to the responses as being due to excitation of descending motor pathways. At the present time, these structures can not be activated by the more widely used magnetic stimulators. ${ }^{1.2}$

With this method we estimated the conduction velocity of the descending motor tracts to be $68 \pm 5 \mathrm{~m} / \mathrm{s}$. This velocity is only approximate because it is based on average lengths of the pathways in postmortem specimens and on the assumption that activation occurs beneath the stimulating cathode. In addition, the value refers only to the fastest conducting fibers within those 
Table 2. Conduction times for the various diseases.

\begin{tabular}{|c|c|c|c|c|c|c|}
\hline 1 & Friedreich's ataxia & $\uparrow$ & $\uparrow$ & $\uparrow$ & $\uparrow$ & $\mathrm{N}$ \\
\hline 2 & Adrenoleukodystrophy & $\uparrow$ & $\uparrow$ & $\uparrow$ & $\uparrow$ & $\mathrm{N}$ \\
\hline 4 & OYL (T4) & $\mathrm{N}$ & $N$ & $\uparrow$ & $\mathrm{N}$ & $\mathrm{N}$ \\
\hline 5 & Arachnoid cyst (T4) & $\mathrm{N}$ & $\mathrm{N}$ & $\uparrow$ & $\mathrm{N}$ & $\mathrm{N}$ \\
\hline 8 & Multiple sclerosis & NR & NR & NR & NR & $\mathbf{N}$ \\
\hline 9 & Spastic paraparesis & $\mathrm{N}$ & $\mathrm{N}$ & $\mathrm{N}$ & $\mathrm{N}$ & $\mathrm{N}$ \\
\hline 10 & Spastic paraparesis & $\mathrm{N}$ & $\mathrm{N}$ & $\mathrm{N}$ & $\mathrm{N}$ & $\mathrm{N}$ \\
\hline 11 & Spastic paraparesis & $\uparrow$ & $\uparrow$ & $\uparrow$ & $\uparrow$ & $\mathrm{N}$ \\
\hline 12 & Spastic paraparesis & $\uparrow$ & $\uparrow$ & $\uparrow$ & $\uparrow$ & $\mathrm{N}$ \\
\hline 13 & Spastic paraparesis & $\uparrow$ & $\uparrow$ & $\uparrow$ & $\uparrow$ & $\mathrm{N}$ \\
\hline
\end{tabular}

OYL: Ossification of the yellow ligament, N: normal, NR: no response, $\uparrow:$ prolonged

pathways and may not be a representative figure for the majority of tracts involved. Nevertheless, we could show significant slowing of conduction in the descending motor tracts of patients with diffuse involvement (Patients 1, 2. 11-14. This slow conduction in patients can be accounted for by the following two possibilities: (i) the maximum conduction velocity of the descending motor tracts through which the responses normally are produced is decreased, or (ii) in patients the stimulus activates different, slower conducting tracts than in controls.

Stimulation of patients with a localized lesion showed localized conduction delay at comparable segments, or delay at all levels caudal to the damage. Conduction delay caudal to the lesion may be caused by Wallerian degeneration of the descending tract which is secondary to the primary involvement. The

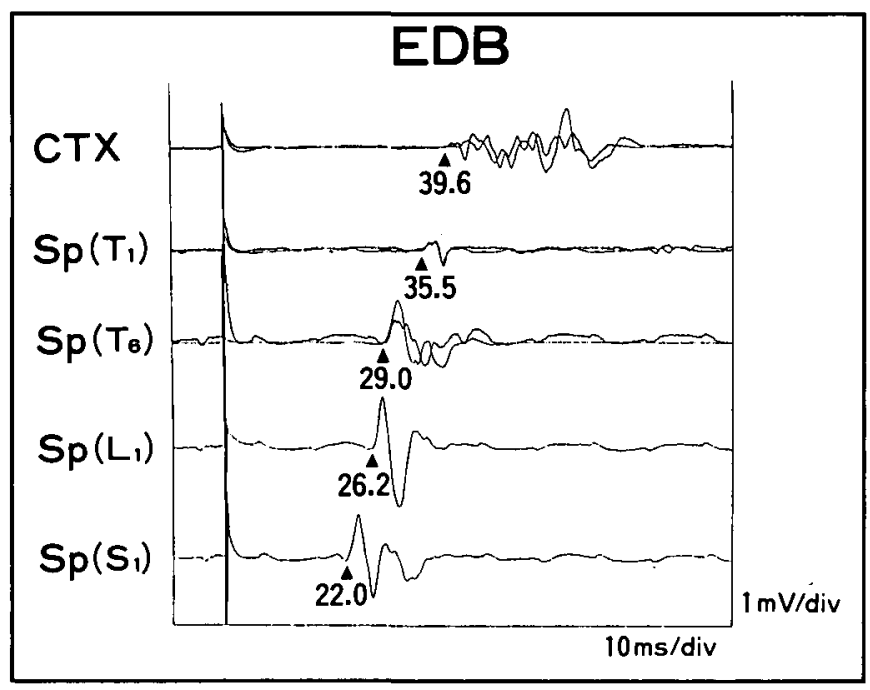

Figure 5: Motor evoked potentials from the EDB of a patient with localized spinal cord compression caused by an ossified yellow ligament at the fourth thoracic vertebral level (Patient 4).

Responses were elicited by stimulation at all the sites. Only the Sp[Tl]$\mathrm{Sp}[\mathrm{T6}] \mathrm{CT}$ is prolonged.

Abbreviations are the same as in Figure 1. technique reported here provides a means of estimating the spinal level of a lesion.

We have calculated the spinal delay time from the time difference between two regression lines at the spinal segment. This value includes synaptic delay and utilization time in active muscles of normal subjects. Average spinal delay time in normal subjects was $0.5 \pm 0.3 \mathrm{~ms}$ (mean $\pm \mathrm{SD}$ ). Assuming that utilization time is some $0.2 \mathrm{~ms}$, synaptic delay is estimated to be at most $0.9 \mathrm{~ms}(\mathrm{p}<0.05)$. This suggests that the descending motor tracts stimulated with this method have a mono- or oligosynaptic connection to motoneurons. Spinal delay time was abnormally prolonged in some patients. This prolongation may result from either: (i) temporal summation of pathologically small and dispersed excitatory post synaptic potentials (EPSPs) or (ii) involvement of polysynaptic pathways not normally used in control subjects.

MRI scans are now a powerful tool for detecting intrinsic spinal cord lesions in clinical practice. Our study showed that our technique revealed lesions in the spinal cord which could not be detected by MRI scan (Patients 1, 2, 11-14). Showing such abnormalities in the spinal cord is one of the values of this technique. This method will not be widely used because of pain induced by electrical stimulation. However, it could be useful in a limited number of cases when functional localization of a spinal cord lesion is critical for treatment or when the MRI scan shows no lesion in the spinal descending motor tracts even though clinical signs strongly suggest a spinal cord lesion. Large stimulating electrodes must be used in such cases because, as we have noted, they reduce the induced pain.

\section{ACKNOWLEDGEMENTS}

The authors are very grateful to Dr. J.C. Rothwell (MRC human movement \& balance unit, Queen Square, London) for his kind revision of this article and for his helpful advice.

\section{REFERENCES}

1. Murray NMF. The clinical usefulness of magnetic cortical stimulation. Electroencephalogr Clin Neurophysiol 1992; 85: 81-85. 
2. Ugawa Y, Rothwell JC, Day BL, Thompson PD, Marsden CD. Magnetic stimulation over the spinal enlargements. J Neurol Neurosurg Psychiatry 1989; 52: 1025-1032.

3. Ugawa Y, Rothwell JC, Day BL, Thompson PD, Marsden CD. Percutaneous electrical stimulation of corticospinal pathways at the level of the pyramidal decussation in humans. Ann Neurol 1991; 29: 418-427.

4. Ugawa Y, Genba K, Mannen T, Kanazawa I. Stimulation of corticospinal pathways at the level of the pyramidal decussation in neurological disorders. Brain 1992; 115: 1947-1961.

5. Snooks SJ, Swash M. Motor conduction velocity in the human spinal cord: slowed conduction in multiple sclerosis and radiation myelopathy. J Neurol Neurosurg Psychiatry 1985; 48: 1135-1139.

6. Ugawa Y, Genba K, Shimpo T, Mannen T. Physiologic analysis of central motor pathways - simultaneous recording from multiple relaxed muscles. Eur Neurol 1989; 29: 135-140.
7. Maertens de Noordhout A, Rothwell JC, Thompson PD, Day BL, Marseen CD. Percutaneous electrical stimulation of lumbosacral roots in man. J Neurol Neurosurg Psychiatry 1988; 51: 174-181.

8. Kimura J. F-wave velocity in the central segment of the median and ulnar nerves: a study in normal subjects and patients with Charcot-Marie-Tooth disease. Neurology 1974; 24: 539-546.

9. Donaldson HH, Davis DJ. A description of charts showing the areas of the cross section of the human spinal cord at the level of each spinal nerve. J Comp Neurol 1903; 13: 19-40.

10. Shimada Y. Brain and spinal cord in Japanese. In: Jinruigakusenshikoza, Tokyo: Youhikaku, 1939; vol 3, 1-188 (in Japanese).

11. DeJong RN. The Neurologic Examination. Hagerstown, Harper \& Row, 1979. 